This document is published in:

Mechanics of Materials, 2013, 60, 107-120. Doi:

http://dx.doi.org/10.1016/j.mechmat.2013.01.009.

(c) Elsevier 


\title{
On the complete extinction of selected imperfection wavelengths in dynamically expanded ductile rings
}

\author{
J.A. Rodríguez-Martínez*, G. Vadillo, R. Zaera, J. Fernández-Sáez \\ Department of Continuum Mechanics and Structural Analysis, University Carlos III of Madrid, Avda. de la Universidad, 30,28911 Leganés, Madrid, Spain
}

Keywords:

Ring expansion

Dynamic necking

Inertia

Stress multiaxiality

Numerical simulations

Stability analysis

\begin{abstract}
A B S T R A C T
In this work the inception and development of multiple necks in dynamically expanded ductile rings with $a b$ initio geometric imperfections has been addressed. Finite element simulations and linear perturbation analysis have been applied for that task. In the numerical calculations a selected wavelength is included into the model defining along the circumference of the ring an array of periodic geometric imperfections of predefined amplitude. In the stability analysis a perturbation of a given mode is added to the background solution and the growth rate of the perturbation is evaluated. The attention has been focused on the extinction of both long and short wavelength imperfections and the appearance of a dominant necking pattern which emerges when the geometric imperfections are vanished. The role played by the loading rate on the extinction of imperfections is also addressed. Moreover, the necking strain is found to be dependent on the imperfection pattern and the loading rate. Its maximum value is registered for the loading cases in which the initial imperfections distribution is completely extinguished.
\end{abstract}

\section{Introduction}

Multiple necking and fragmentation occurring in ductile materials subjected to high loading rates have attracted the interest of researchers over the years. This material failure mode is observed in engineering applications like ballistic impact, car crashes, blast protection or orbital debris impact on spacecraft structures.

In the last decades a number of laboratories have developed specific experimental arrangements with the aim of characterizing dynamic necking and fragmentation of solids. Most of these experimental configurations rely on the radial expansion of axially symmetric structures like rings (Niordson, 1965; Grady and Benson, 1983; Gourdin, 1989; Altynova et al., 1996; Grady and Olsen, 2003; Zhang and Ravi-Chandar, 2006; Janiszewski, 2012), tubes (Mott, 1947; Wesenberg and Sagartz, 1977; Goto et al., 2008; Hiroe et al., 2008; Zhang and Ravi-Chandar, 2010) and hemispheres (Juanicotena, 1998; Mercier et al., 2010) since

\footnotetext{
* Corresponding author. Tel.: +34 916248460; fax: +34 916249430.

E-mail address: jarmarti@ing.uc3m.es (J.A. Rodríguez-Martínez).
}

complications resulting from wave propagation are eliminated due to the symmetry of the problem, which facilitates the interpretation of the experimental findings. In these tests the material stretches during loading until homogeneous deformation fails at large strain, leading to flow localization in the form of multiple necking and subsequent fragmentation. Within the typical range of loading velocities attained in these tests, tipically from 50 to $300 \mathrm{~m} / \mathrm{s}$, the experimental results show that the strain to failure of ductile materials is enhanced by the expansion velocity (Hu and Daehn, 1996; Altynova et al., 1996; Janiszewski, 2012). The failure pattern is revealed velocity dependent too, leading to an increasing number of necks and fragments with testing velocity (Grady and Benson, 1983; Zhang and Ravi-Chandar, 2006).

Beside his famous experiments, Mott (1947) opened the theoretical field of modelling multiple necking and fragmentation processes. Mott postulated that fragmentation proceeds through the random spatial and temporal occurrence of fractures resulting in a distribution of fragment lengths. The fragment size distribution was related to the statistical variability in the failure strain of the material 
and was obtained through an estimate of the propagation of the release waves from each fracture event. The pioneering work of Mott (1947) have received continuous attention over the years and a number of publications have attempted to provide further validation to the statistics-based theory (Zhang et al., 1999; Elek and Jaramaz, 2005; Elek and Jaramaz, 2008). Within this framework, it is worth noting the works of Grady and co-workers (Grady, 1981; Kipp and Grady, 1985; Grady and Olsen, 2003) who extended the original developments of Mott to provide explicit expressions for the time to fracture and the fracture strain.

Bifurcation and stability analyses have also been conducted to model multiple necking and fragmentation. The starting point was the pioneering work of Hill and Hutchinson (1975) who developed a quasi-static bifurcation analysis for a rectangular plate strained under plane strain tension. They reported that if inertia effects are neglected, short wavelength modes could not be developed and long wavelength modes were boosted. Somewhat later, in a series of celebrated papers, Molinari and co-workers (Fressengeas and Molinari, 1994; Mercier and Molinari, 2003; Mercier and Molinari, 2004) provided further verification to previous idea and showed that the multiaxial character of the stress state in a necked region has a damping effect on short wavelength modes. Furthermore, they revealed the damping effect of inertia on long wavelength modes (Fressengeas and Molinari, 1985, 1994). This was later confirmed in the works due to Sørensen and Freund (1998) and Shenoy and Freund (1999). It was concluded that short wavelengths are stabilized by stress multiaxiality effects and long wavelengths by inertia, promoting an intermediate wavelength which determines the spacing between localization points in the multiple necking process (Mercier and Molinari, 2003; Mercier and Molinari, 2004; Zhou et al., 2006; Vadillo et al., 2012).

Further advances in understanding the mechanisms which reside behind multiple necking and fragmentation processes were made with the advent of computational mechanics. Han and Tvergaard (1995) conducted finite element simulations of the ring expansion problem. A number of long wavelength geometric imperfections were distributed along the circumference of the ring for triggering the inception of necks. The authors reported that multiple necking occurred in the simulations, being the final pattern of necks largely independent of the location of the initial imperfections, when their amplitudes were small enough. Moreover, Tuğcu (1996) analysed via numerical simulations the dynamic response of cylindrical shells subjected to internal pressure. The necks inception was triggered by imposing geometric imperfections of two different mode shapes on the initial state. After evaluation of the growth rate of the deformation mode it was concluded that the mode with the shorter wavelength developed sooner and faster than the one with the larger wavelength. In a posterior work, Tuğcu (2003) provided deeper insight in the numerical simulation of the dynamic expansion of cylindrical shells. A single long wavelength imperfection was prescribed along the circumference of the shell. It was reported that, alternatively, localization at the initial imperfection site or multiple necking may be generated. The result depending on the current combination of geometric, material and loading parameters. Within the computational works devoted to the role played by geometric imperfections on necking inception, the paper due to Sørensen and Freund (2000) has to be highlighted. The authors performed FE analysis of thin rings at high radial rate expansion specifically addressing the influence of the imperfection wavelength on multiple necking formation. It was reported that long wavelength imperfections did not act as neck precursor sites. Instead, a critical, nearly periodic mode with short wavelength appears at rather large overall strain levels. Neck spacing was seen to have little correlation with the initial imperfections. Thus, the works by Han and Tvergaard (1995), Tuğcu (1996, 2003) and Sørensen and Freund (2000) provided verification via finite elements of the role played by inertia in damping long wavelength imperfections; which fully agrees with the observations yielded from the stability analyses aforementioned in previous paragraph. Thus, progress in computational mechanics have allowed a better understanding of multiple necking and fragmentation processes occurring in ductile materials subjected to dynamic loadings. However, there is a number of issues that need further numerical investigations, the following are addressed in this paper:

- In agreement with stability analyses results, finite element computations show that inertia extinguishes sufficiently long wavelength imperfections. So, in agreement with stability analyses results, may finite element computations corroborate that stress multiaxiality effects extinguish sufficiently short wavelength imperfections?

- If this is so, does the loading velocity play a role on the process of extinction? Furthermore, does the loading velocity affect the imperfection wavelengths that are suppressed by inertia and stress multiaxiality effects?

- Moreover, is the necking strain of the material affected by the presence of imperfections? If this is so, does the necking strain affect the imperfection wavelengths that are suppressed by inertia and stress multiaxiality effects?

In this work, the previous issues are approached relaying on 3D finite element simulations of the ring expansion problem. A selected wavelength is included into the model defining along the circumference of the ring an array of periodic geometric imperfections of predefined amplitude. Then, numerical computations are conducted over wide ranges of loading rates and imperfection wavelengths in order to demonstrate that both sufficiently short and long wavelengths can be suppressed by the stabilizing effects of stress multiaxiality and inertia. Then, the effects of imperfection geometry, loading rate and necking strain in the process of extinction are analysed. The results are further rationalized relying on a linear stability analysis.

\section{Three dimensional finite element modeling}

Similarly to Rusinek and Zaera (2007) a Lagrangian 3D finite element model of the rapid expansion of ductile rings 
has been developed. Additionally, a selected wavelength has been included into the model defining an array of equally-spaced geometric imperfections of predefined amplitude. For that task, a variable and periodic radial thickness was considered in the reference configuration $(t=0)$. The outer radius was defined by a harmonic function and the inner radius was kept constant, similarly to Han and Tvergaard (1995), Tuğcu (1996) and Sørensen and Freund (2000). The domain of the ring is given in cylindrical coordinates by the following equations:

$$
\begin{aligned}
& R_{\text {int }} \leqslant r \leqslant R_{\text {ext }}-\frac{\delta}{2}(1-\cos (N \theta)) \\
& 0 \leqslant \theta \leqslant 2 \pi \\
& -\frac{h}{2} \leqslant z \leqslant \frac{h}{2}
\end{aligned}
$$

$R_{\text {int }}$ being the inner radius, $R_{\text {ext }}$ the maximum outer radius, $\delta$ the amplitude of the imperfection, $N$ the number of imperfections and $h$ the thickness of the ring in direction $z$, see Fig. 1. The wavelength of the imperfection is then defined by $W_{i m p}=2 \pi R_{\text {int }} / N$. Table 1 shows the values adopted for these dimensions, in consonance with those proposed by Rusinek and Zaera (2007).

The loading condition is a radial velocity, $V_{r}$, applied on the inner surface of the ring, which remains constant throughout the entire process (Rusinek and Zaera, 2007; Vadillo et al., 2012; Rodríguez-Martínez et al., 2013b). The initial condition is a radial velocity of the same value $V(t=0)=V_{r}$ applied to all the nodes of the model. It should be noted that application of this initial condition is essential in order to avoid spurious propagation of waves through the thickness of the ring resulting from the abrupt motion of the inner face at $t=0$ while the reminder of the ring is initially at rest. Otherwise, for

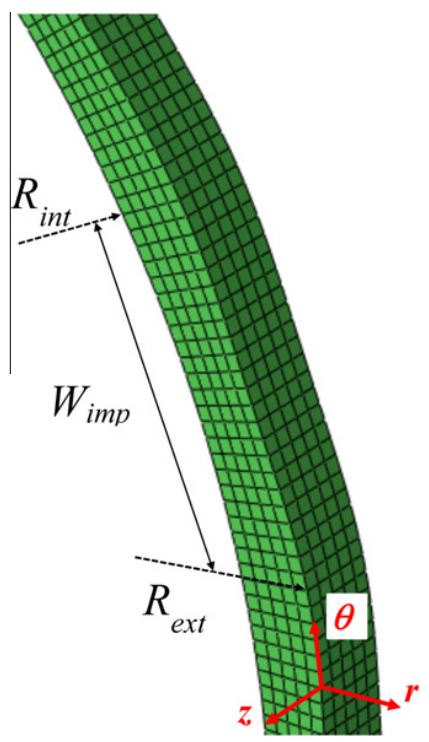

Fig. 1. Mesh of a ring sector corresponding to an imperfection wavelength $(N=20)$. A large imperfection amplitude $\delta$ has been shown in the figure, $\delta=\left(R_{\text {ext }}-R_{\text {int }}\right) / 10$, for better illustration of the geometric perturbation.
Table 1

Dimensions of the ring.

\begin{tabular}{llll}
\hline$R_{\text {int }}(\mathrm{mm})$ & $R_{\text {ext }}(\mathrm{mm})$ & $\delta(\mathrm{mm})$ & $h(\mathrm{~mm})$ \\
\hline 15 & 16 & $10^{-2}$ or $5 \cdot 10^{-2}$ & 1 \\
\hline
\end{tabular}

sufficiently high velocities, the generated plastic wave itself could induce a neck (Needleman, 1991; Xue et al., 2008). The initial strain rate is given by $\dot{\varepsilon}_{0}=V_{r} / R_{\text {int }}$. Huber-Mises ideal plasticity has been assumed to describe the material response, setting aside strain or strain rate hardening phenomena. The parameters defining the material behavior are representative of steel: $E=$ $200 \mathrm{GPa}, v=0.3, \sigma_{y}=500 \mathrm{MPa} \quad$ and $\rho=7800 \mathrm{~kg} / \mathrm{m}^{3}$. This material description allows proper determination of the mechanisms responsible for the extinction of selected imperfection wavelengths in the radial expansion of ductile rings, as it will be shown in forthcoming sections of the paper.

The solution of the initial boundary value problem was obtained using the ABAQUS/Explicit 6.10 (Simulia, 2010) finite element code, well-suited to simulate high-speed, nonlinear, transient dynamic events. The domain is mapped to cartesian coordinates and meshed using eight-node tri-linear brick elements with reduced integration, C3D8R in ABAQUS notation. The viscous method available in ABAQUS/Explicit has been used to prevent hourglass deformation modes; the scale factor used for all hourglass stiffnesses was chosen equal to one.

A mesh convergence study has been performed; the time evolution of different critical output variables, namely stress, strain and necking strain, were compared against a measure of mesh density until the results converged satisfactorily. According to the considerations reported by $\mathrm{Zu}-$ kas and Scheffer (2000) the aspect ratio of the elements was close to $1: 1: 1\left(\approx 0.2 \times 0.2 \times 0.2 \mathrm{~mm}^{3}\right)$. This element size also ensures 4 elements along the shortest imperfection wavelength considered in the work. In radial direction a bias meshing factor was used to keep constant the radialto-hoop length ratio of the elements. Fig. 1 shows the mesh of a ring sector corresponding to the imperfection wavelength. The code uses an explicit scheme, the central difference operator, which is only conditionally stable, i.e. the time step must be small enough to assure that the solution does not grow unbounded. The stability limit for the operator is inversely proportional to the highest natural frequency in the system (Plěsek et al., 2010). Additionally a small amount of damping is introduced by the code, reducing the stability limit to control high frequency oscillations.

The whole finite element model was defined with a script to allow a rapid change of the imperfection wavelength and amplitude.

\section{Linear perturbation analysis}

The equations governing the stretching process of the expanding ring are derived within a quasi-1D theoretical framework. Details of the formulation can be found in Zhou et al. (2006) and Vadillo et al. (2012), but we present here the main equations for completeness. The ring 
expansion process is approximated to a cylindrical bar with cross-section radius $r_{0}$, area $A_{0}=\pi r_{0}^{2}$, and length $L$ subjected to axial velocity. The fundamental equations of the loading process are presented below:

$$
\left\{\begin{array}{l}
\left(\frac{\partial v}{\partial X}\right)_{t}=e^{\varepsilon} \dot{\varepsilon} \\
\rho A_{0}\left(\frac{\partial v}{\partial t}\right)_{X}=A\left(\frac{\partial \sigma}{\partial X}\right)_{t}+\sigma\left(\frac{\partial A}{\partial X}\right)_{t} \\
A=A_{0} e^{\varepsilon} \\
\sigma_{y}=\sigma_{0} \\
\sigma=\left(1+\theta^{-1}\right) \ln (1+\theta) \sigma_{y} \\
\theta=\frac{1}{2} r\left(\frac{\partial^{2} r}{\partial x^{2}}\right)=\frac{2 A\left(\partial^{2} A / \partial x^{2}\right)-(\partial A / \partial x)^{2}}{8 \pi A}
\end{array}\right.
$$

where, at time $t$ and for any cross section of the bar, $X$ is the Lagrangian coordinate $X(0 \leqslant X \leqslant L), x$ is the Eulerian coordinate, $v$ the current axial velocity, $r$ and $A$ the current radius and cross-sectional area, respectively, and $\sigma$ the true stress. Moreover $\varepsilon=\ln \left[\left(\frac{\partial x}{\partial X}\right)_{t}\right], \dot{\varepsilon}=(\partial \varepsilon / \partial t), \sigma_{y}$ is the material yield stress and $\theta$ is a geometrical parameter which gathers the stress multiaxial effects tied to necked section (Bridgman, 1952; Walsh, 1984; Fressengeas and Molinari, 1985). The initial and boundary conditions are $v(X, 0)=\dot{\varepsilon}_{0} X, v(0, t)=0$ and $v(L, t)=\dot{\varepsilon}_{0} L$. Then, the system of Eqs. (2) has an homogeneous solution of the type $f_{1}=\left(v_{1}(X), \varepsilon_{1}(t), \dot{\varepsilon}_{1}(t), A_{1}(t), r_{1}(t), \sigma_{1}(t), \theta_{1}\right)^{T}$ which defines the background state of the boundary value problem in absence of flow instability.

At a time $t_{0}$ a perturbation of the form $\delta f e^{i \xi X}=(\delta v, \delta \varepsilon, \delta \dot{\varepsilon}, \delta A, \delta r, \delta \theta, \delta \sigma)^{T} e^{i \xi X}$ is superimposed to the fundamental solution (Zhou et al., 2006; Vadillo et al., 2012). $\xi$ is the wavenumber and $(\delta v, \delta \varepsilon, \delta \dot{\varepsilon}, \delta A, \delta r, \delta \theta, \delta \sigma)$ are the differences between the actual perturbed solution and the homogeneous solution. Then, at a time $t>t_{0}$ the perturbed solution of the aforementioned system of equations has the form $f=f_{1}+\delta f e^{i \xi X+\eta\left(t-t_{0}\right)}$ where $\eta$ is the perturbation grow rate. By substituting previous expression into Eq. (2) and keeping only the first-order terms, a linear homogeneous system to obtain $\delta f$ is derived.

A non-trivial solution for $\delta f$ can be found if the determinant of the coefficient matrix of the system of algebraic linear equations is equal to zero. This leads to the following quadratic expression in $\eta$

$\eta^{2}+\dot{\varepsilon}_{1}\left(t_{0}\right) \eta+\frac{\xi^{2}}{\rho_{0}} e^{-2 \varepsilon_{1}\left(t_{0}\right)}\left[\sigma_{1}\left(t_{0}\right)\left(\frac{A_{0} e^{-3 \varepsilon_{1}\left(t_{0}\right)}}{8 \pi} \xi^{2}-1\right)\right]=0$

At this point, the following dimensionless variables are introduced (Zhou et al., 2006)

$\bar{\eta}=\frac{\eta}{\dot{\varepsilon}_{1}} \quad \bar{\xi}=r_{0} \xi \quad \bar{L}=\frac{\sqrt{\sigma_{0} / \rho}}{r_{0} \dot{\varepsilon}_{1}}$

where $\bar{\eta}$ is the dimensionless perturbation growth, $\bar{\xi}$ is the dimensionless wavenumber and $\bar{L}$ is a dimensionless parameter such that $1 / \bar{L}^{2}$ represents the inertial resistance to motion. It should be noted that inertia accounts for the intrinsic effects that material density, sample dimensions, flow stress level and loading rate all have on necking inception as described elsewhere (Knoche and Needleman, 1993; Mercier and Molinari, 2003; Mercier and Molinari, 2004; Vadillo et al., 2012). The material behaviour is stabilized as the dimensionless parameter $\bar{L}$ decreases, delaying the formation and development of necks.

Then, with application of the dimensionless variables, Eq. (3) takes the following form

$\bar{\eta}^{2}+\bar{\eta}+\bar{L}^{2} \Lambda^{2} \bar{\xi}^{2}\left(\Lambda^{3} \frac{\bar{\xi}^{2}}{8}-1\right)=0$

where $\Lambda=e^{-\varepsilon_{1}\left(t_{0}\right)}$.

In generalized form, the condition for the perturbation growth is $\bar{\xi}<\frac{2}{\Lambda} \sqrt{\frac{2}{\Lambda}}$. If this condition is fulfilled the perturbation growth is defined by

$\bar{\eta}^{+}=\frac{-1+\sqrt{1+4 \bar{L}^{2} \Lambda^{2} \bar{\xi}^{2}\left(1-\Lambda^{3} \frac{\bar{\xi}^{2}}{8}\right)}}{2}$

The perturbation growth is assumed to represent the onset of diffuse necking, the very first stages in which the plastic flow deviates from the background deformation. Note that, in contrast with the FE model, here the perturbation is not representative of any particular geometric entity but it accounts for any lack of homogeneity existing in the variables of the problem which may lead to flow localization.

\section{Analysis and results}

Results of the finite element computations and stability analysis are presented. Following the terminology of Sørensen and Freund (2000) the necking process will be described as stable if a single neck forms in each site where the wall thickness is minimum. On the other hand, the necking process will be described as unstable if necks form at sites other than the locations of minimum wall thickness. Note that numerical calculations are run within wide ranges of loading rates $667 \mathrm{~s}^{-1} \leqslant \dot{\varepsilon}_{0} \leqslant 66667 \mathrm{~s}^{-1}$, corresponding to $10 \mathrm{~m} / \mathrm{s} \leqslant V_{r} \leqslant 1000 \mathrm{~m} / \mathrm{s}$.

\subsection{The effect of imperfection geometry on necking formation}

According to Eq. (1), the imperfection geometry is defined through the parameters $N$ - number of imperfections - and $\delta$ - amplitude of the imperfection. In this section, the influence of each of them on the multiple necking process will be considered separately.

\subsubsection{The effect of imperfection spacing}

Fig. 2(a) illustrates the number of necks formed as a function of the number of initial imperfections in numerical computations carried out at $\dot{\varepsilon}_{0}=33333 \mathrm{~s}^{-1}$ for $\delta=1 \%$. The attention will be focussed on the interplay between the number of imperfections introduced in the FE model $N$ and the number of necks incepted $n$. Three main situations can be identified:

- Region I - Long imperfection wavelengths: the number of necks incepted is independent of the imperfections spacing and a dominant necking pattern insensitive to the geometric perturbations is identified as shown in Fig. 2(a). At the onset of loading, non-localized excursions of strain appear at the sites of the minimum wall thickness growing at rates comparable to the rate of the background deformation as illustrated in Fig. 3(a), 


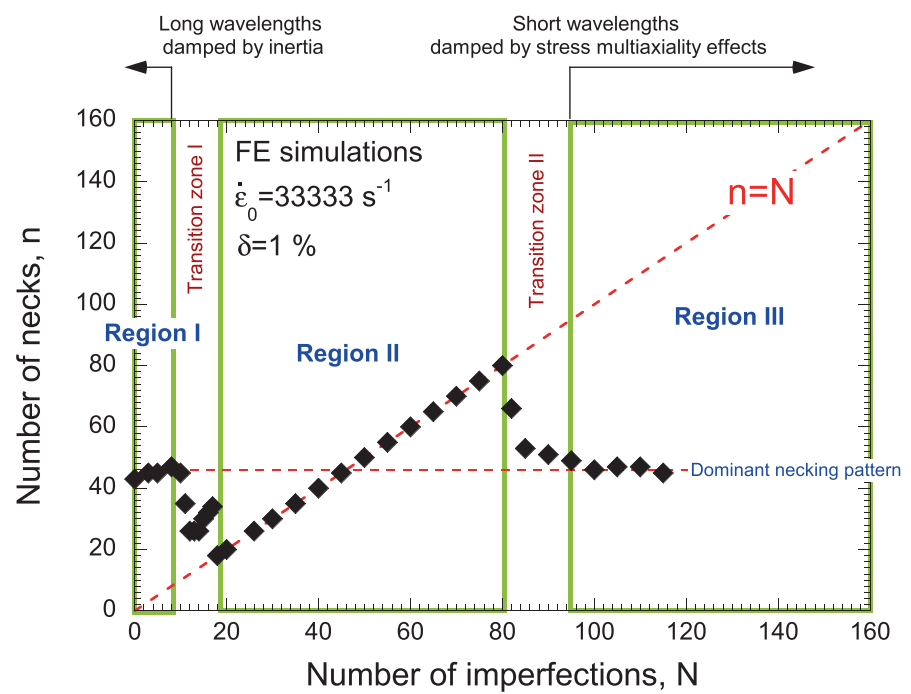

(a)

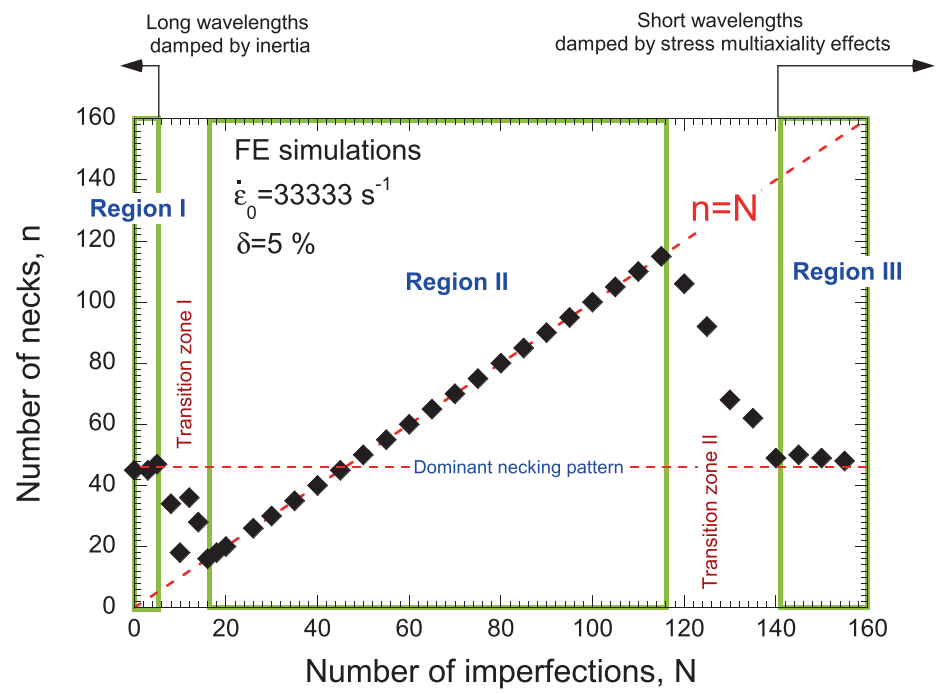

(b)

Fig. 2. Finite element results. Number of necks $n$ as a function of the number of imperfections $N$ for $\dot{\varepsilon}_{0}=33333 \mathrm{~s}^{-1}$ and two different imperfection amplitudes. (a) $\delta=1 \%$, (b) $\delta=5 \%$.

where the equivalent plastic strain $\bar{\varepsilon}^{p}$ is depicted upon (half) of the normalized ring perimeter $\bar{P}$. Later in the deformation, a cascade of (almost) equally-spaced localized necks - so called because further deformation of the ring concentrates in these localized regions - are formed (practically) simultaneously all along the ring as shown in Fig. 3(a). The locations of these necks do not correlate with the initial imperfections distribution, i.e. the necking process is unstable. Region I is controlled by the stabilizing effect that inertia has on long wavelength perturbations as reported by Fressengeas and Molinari (1985).

- Region II - Intermediate imperfection wavelengths: each geometric imperfection leads to the nucleation of a single neck as illustrated in Fig. 2(a). At very early stages of loading, localized necks (equally-spaced and equally-intense) appear at the sites of the minimum wall thickness growing at much faster rates than that of the background deformation as can be observed in Fig. 3(b). The necking process is stable. Region II is characterized by the enhancing effect that intermediate wavelengths have on necking inception as reported by Mercier and Molinari (2003), Mercier and Molinari (2004).

- Region III - Short imperfection wavelengths: the number of necks incepted is independent of the imperfections spacing and a dominant necking pattern insensitive to the geometric perturbations is identified as illustrated Fig. 2(a). The process of necking nucleation is analogous to that described for region I; at the 


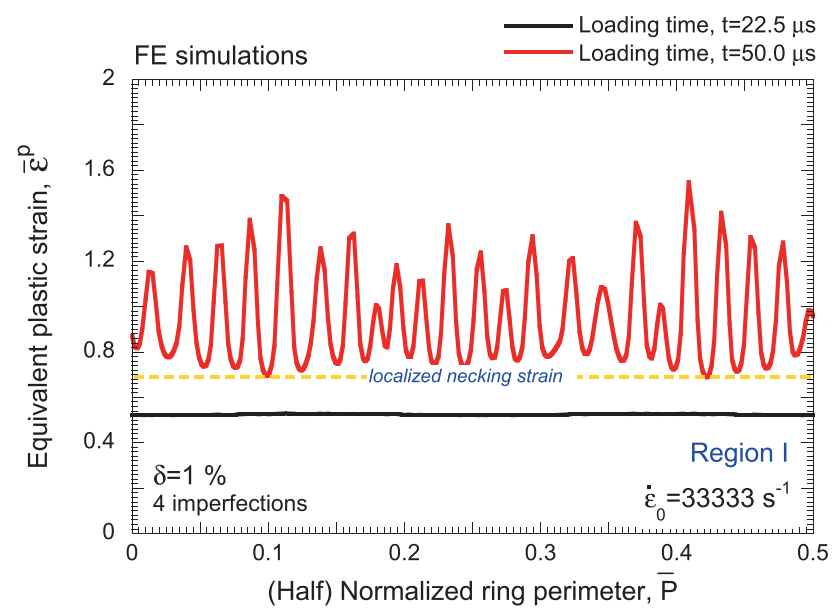

(a)

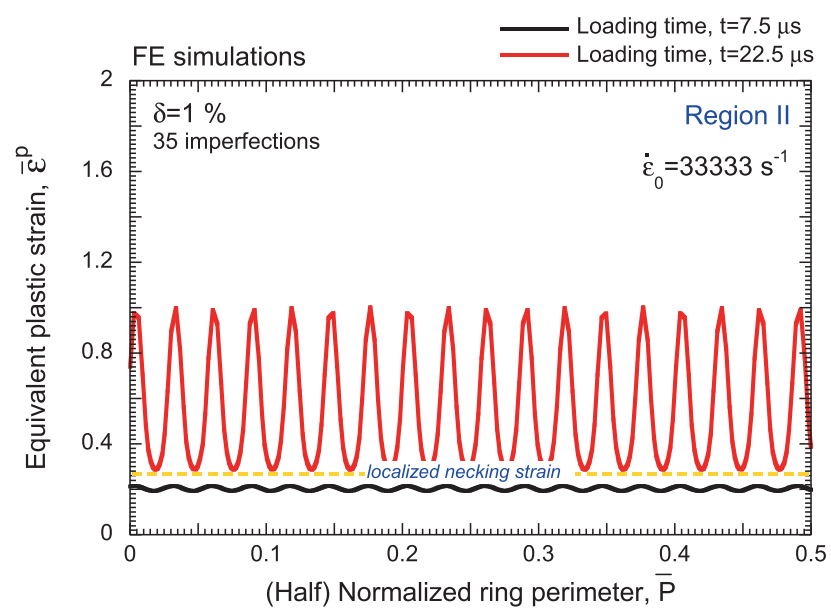

(b)

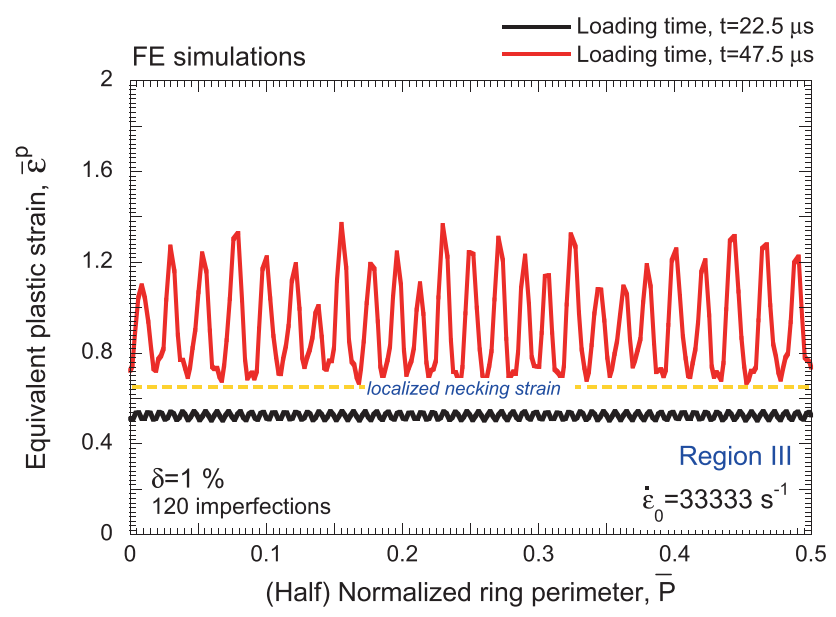

(c)

Fig. 3. Finite element results. Equivalent plastic strain $\bar{\epsilon}_{p}$ along (half) the normalized perimeter of the ring $\bar{P}$ for $\dot{\varepsilon}_{0}=33333 \mathrm{~s}^{-1}$ and $\delta=1 \%$. (a) Region I: 4 imperfections, (b) region II: 35 imperfections, (c) region III: 120 imperfections. 
onset of loading non-localized excursions of strain appear at the sites of the minimum wall thickness growing at rates comparable to the rate of the background deformation as reported in Fig. 3(c). As deformation proceeds, an array of (almost) equally-spaced localized necks are formed (almost) simultaneously all along the circumference. The number of necks nucleated coincides with that registered in region I. The locations of these necks do not correlate with the initial imperfections distribution, i.e. the necking process is unstable. Region III is controlled by the stabilizing effect that stress multiaxiality has on short wavelength perturbations as reported by Zhou et al. (2006).

This analysis reveals that for sufficiently long/short wavelengths the stabilizing effect of inertia/stress multiaxiality completely vanishes the effect of geometric imperfections. Such behaviour reveals a dominant necking pattern which characterizes the loading process.

Furthermore, it should be noted that transition zones between regions I, II and III can be identified in Fig. 2(a). These are zones in which the necking process can not be strictly defined as stable or unstable. Within these transition zones the loading process involves formation of necks of both types. Neither the number of necks incepted is independent of the imperfections distribution nor the number of necks incepted coincides with the number of imperfections.

- Transition zone I: corresponds to a situation between regions I and II, see Fig. 2(a). Localized excursions of strain appear in the locations in which the ring wall is initially thinnest. However, as deformation proceeds multiple necks appear in these locations leading to a non-uniform neck spacing as illustrated in Fig. 4(a). In the transition zone I the geometrical imperfections are only partially damped by the stabilizing effect of inertia.

- Transition zone II: corresponds to a situation between regions II and III, see Fig. 2(a). Most necks appear in the locations in which the ring wall is initially thinnest, however some other do not. The neck spacing is not uniform as illustrated Fig. 4(b). In the transition zone II the geometrical imperfections are only partially damped by the stabilizing effect of stress multiaxiality.

\subsubsection{The effect of imperfection amplitude}

Next, the effect of imperfection amplitude on the necking process is addressed. Fig. 2 shows the number of necks formed as a function of the number of initial imperfections in numerical computations conducted for $\dot{\varepsilon}_{0}=33333 \mathrm{~s}^{-1}$ and two different imperfection amplitudes, $\delta=1 \%$ in Fig. 2(a) and $\delta=5 \%$ in Fig. 2(b). Increasing imperfection amplitude decreases the number of imperfections which determine the lower bound of region II and increases the number of imperfections which determines the upper bound of region II, extending the range for which the imperfections distribution leads to stable necking process. In other words, increasing imperfection amplitude hinders overcoming of geometrical perturbations by inertia and stress multiaxiality. Note that as the imperfection amplitude increases the hydrostatic pressure does also.
However, such increase of the stress multiaxiality effects (stabilizing effect) seems to be cancelled due the enhanced stress concentration (destabilizing effect) caused by the associated cross section reduction.

\subsection{The effect of loading rate on necking formation}

Next, the effect of loading rate on the necking process is addressed. The results shown in Fig. 5 illustrate the number of necks $n$ formed as a function of the number of initial imperfections $N$, for $\delta=1 \%$. The numerical computations are carried out at two different initial loading rates: $\dot{\varepsilon}_{0}=16667 \mathrm{~s}^{-1}$ and $\dot{\varepsilon}_{0}=66667 \mathrm{~s}^{-1}$, below and above that considered in Fig. 2(a) respectively. Comparing Fig. 5 with Fig. 2(a) a main effect can be identified: as the loading velocity increases region II is moved to shorter imperfection spacings; its lower and upper bounds both are shifted to larger number of imperfections.

A rational explanation to this finding is provided relying on finite element calculations (to determine the influence of loading rate on the necking inception) and linear stability analysis (to understand the mechanisms responsible for the shifting of region II as the loading rate increases).

\subsubsection{Finite element results}

The localized necking strain $\bar{\varepsilon}_{\text {neck }}^{p}$ has been determined in finite element computations conducted for different loading rates and imperfection spacings. Following Triantafyllidis and Waldenmyer (2004), Xue et al. (2008) and Rodríguez-Martínez et al. (2013b), Rodríguez-Martínez et al. (2013a), the localized necking strain $\bar{\varepsilon}_{\text {neck }}^{p}$ is assumed as determined by the condition $\frac{d \bar{c} p}{d t}=0$, where $\bar{\varepsilon}^{p}$ is the equivalent plastic strain measured within the unloading zone which surrounds the necks and $t$ refers to time, as shown in Fig. 6. Note that the neck spacing $W_{\text {neck }}$ may not coincide in certain loading cases with the imperfection spacing $W_{i m p}$.

Fig. 7 illustrates the localized necking strain upon the number of imperfections for different loading rates. It can be observed that $\bar{\varepsilon}_{\text {neck }}^{p}$ increases with loading rate (Fyfe and Rajendran, 1980; Grady and Benson, 1983; Knoche and Needleman, 1993; Hu and Daehn, 1996), regardless the number of imperfections introduced in the FE model. It is worth noting that the curves $\bar{\varepsilon}_{\text {neck }}^{p}$ versus $N$ display a characteristic shape independently of the loading velocity. Based on this curve-shape, regions I, II and III can be identified. For that task, the attention is focused on the results corresponding to $\dot{\varepsilon}_{0}=16667 \mathrm{~s}^{-1}$ :

- Region I is defined by the largely constant values of $\bar{\varepsilon}_{\text {neck }}^{p}$ measured within the range $N \lesssim 6$, see the agreement with Fig. 5(a). The range of imperfections for which $\bar{\varepsilon}_{n e c k}^{p}$ keeps constant increases with loading rate.

- Region II is defined by the concave up shape displayed by the results measured within the range $6 \lesssim N \lesssim 60$, see the agreement with Fig. 5(a). The decrease of $\bar{\varepsilon}_{\text {neck }}^{p}$ corresponds to loading cases for which the necking process is stable $(n=N)$ but the number of imperfections is lower than the number of necks defined by the dominant necking pattern. The minimum value of $\bar{\varepsilon}_{\text {neck }}^{p}$ is closely related to the number of imperfections which 


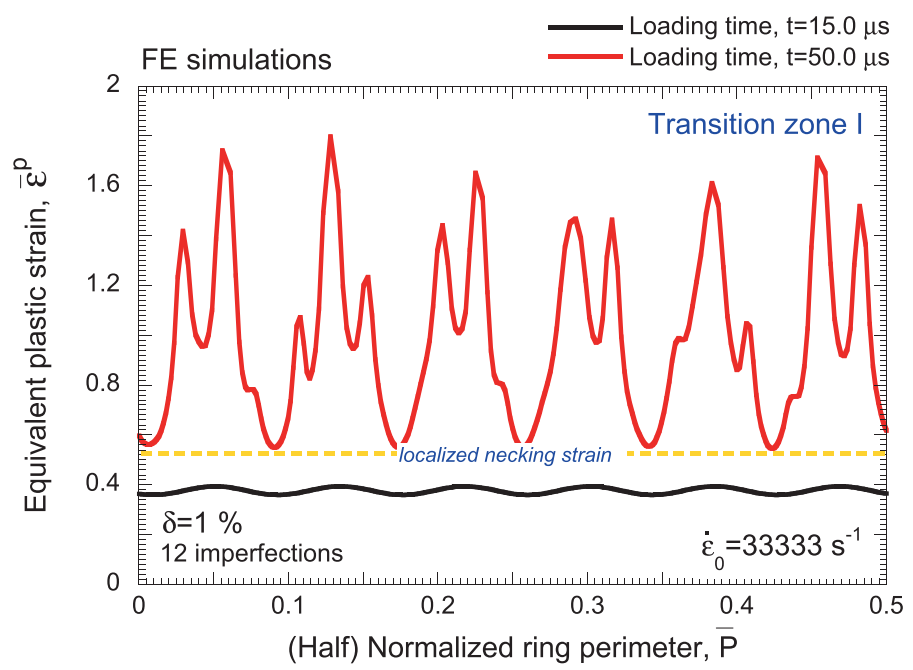

(a)

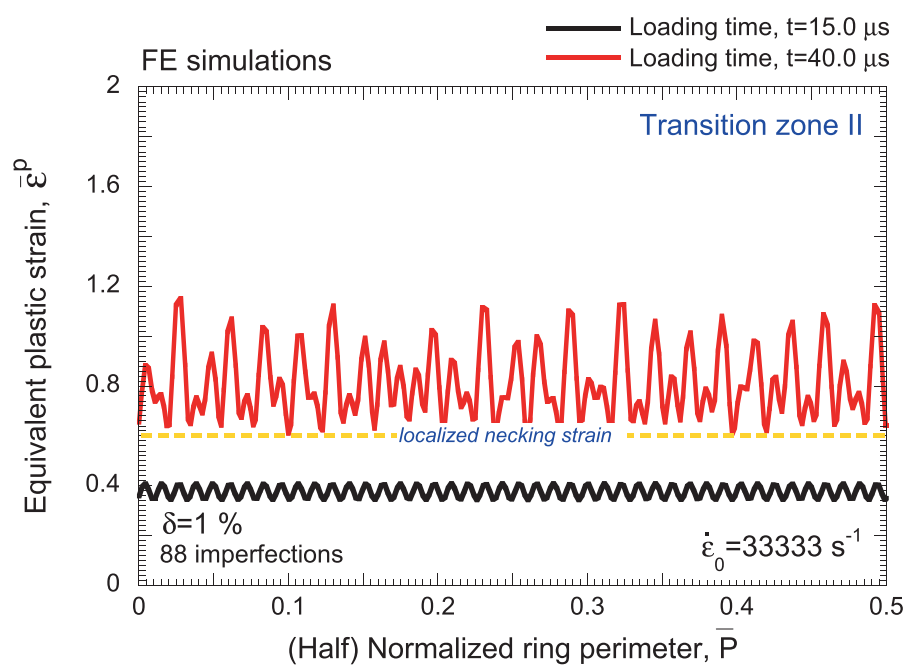

(b)

Fig. 4. Finite element results. Equivalent plastic strain $\bar{\epsilon}_{p}$ along (half) the normalized perimeter of the ring $\bar{P}$ for $\dot{\varepsilon}_{0}=33333 \mathrm{~s}^{-1}$ and $\delta=1 \%$. (a) Transition zone I: 12 imperfections, (b) Transition zone II: 88 imperfections.

coincides with the dominant necking pattern. This agrees with the considerations reported by RodríguezMartínez et al. (2013b) who correlated the dominant necking pattern with the minimum investment of energy required for the necking formation. The increase of $\bar{\varepsilon}_{\text {neck }}^{p}$ corresponds to loading cases for which the necking process is stable but the number of imperfections exceeds that determined by the dominant necking pattern.

- Region III is defined by the largely constant values of $\bar{\varepsilon}_{\text {neck }}^{p}$ measured within the range $N \gtrsim 60$, see the agreement with Fig. 5(a). Note that the range of imperfections for which $\bar{\varepsilon}_{\text {neck }}^{p}$ keeps constant decreases with loading velocity. In fact, for the largest loading rate considered $\dot{\varepsilon}_{0}=66667 \mathrm{~s}^{-1}$, such constancy of the necking strain is hardly achieved for the larger values of imperfections considered, $N \gtrsim 130$, as depicted in Fig. 5(b).

\subsubsection{Linear stability results}

The linear stability analysis permits to evaluate the dimensionless perturbation growth $\bar{\eta}^{+}$upon the number of imperfections $N$ for different background strains $\varepsilon_{1}$ and loading rates $\dot{\varepsilon}_{0}$, according to Eq. (6). Note that the number of periodic imperfections $N$ is related to the dimensionless wavenumber $\bar{\xi}$ through $N=2 \bar{\xi} R_{\text {int }} / h$, where $h$ is used to designate indifferently the width of the (almost) square cross-section in the 3D simulations or the diameter of the circular-cross section in the stability analysis. 


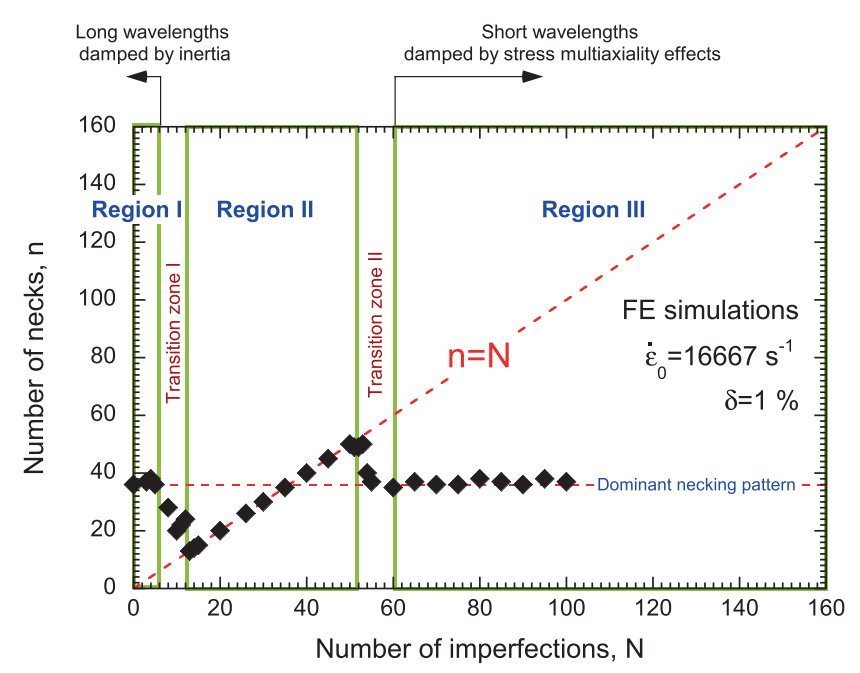

(a)

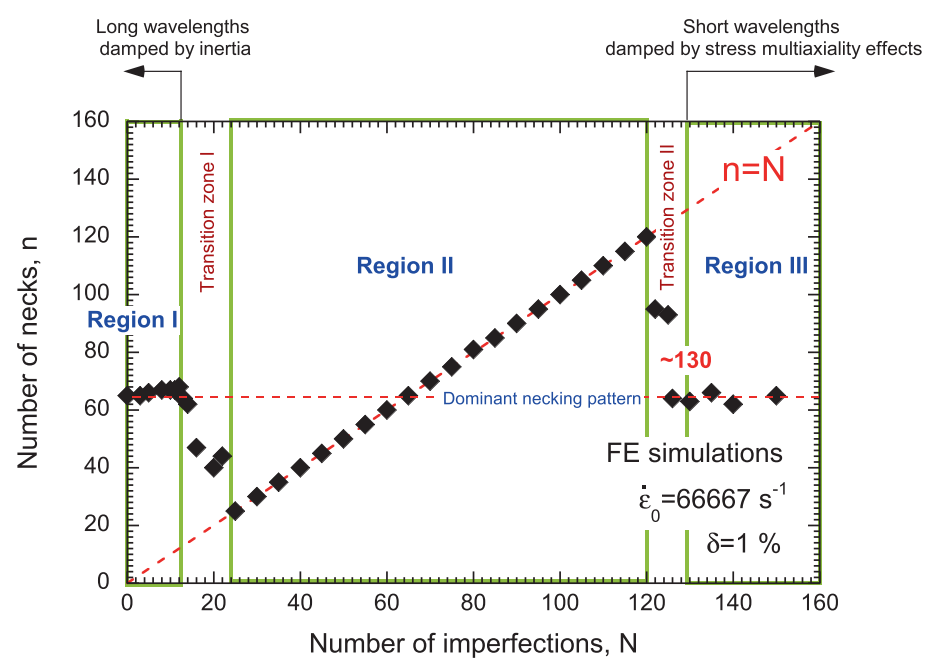

(b)

Fig. 5. Finite element results. Number of necks $n$ as a function of the number of imperfections $N$ for $\delta=1 \%$ and two different initial strain rates. (a) $\dot{\varepsilon}_{0}=16667 \mathrm{~s}^{-1}$, (b) $\dot{\varepsilon}_{0}=66667 \mathrm{~s}^{-1}$.

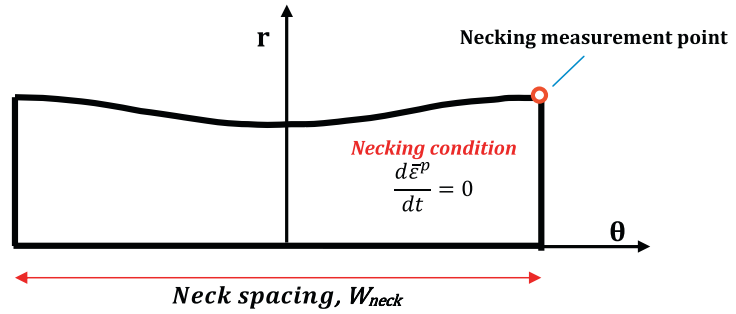

Fig. 6. Schematic representation of a necked zone showing the measurement point used for determination of the localized necking condition $\frac{d \bar{z} p}{d t}=0$ in the finite element computations.

Fig. 8 shows that, for a given value of the background strain, as the loading rate increases the perturbation growth is slowed down for any wavenumber considered.
Fig. 8 also shows that, for a given value of the loading rate, as the background strain increases the promotion of shorter wavelengths is favoured. However, note that a proper analysis cannot consider the effect of background strain and strain rate separately. According to the finite element results, the loading rate increase is accompanied by the localized necking strain increase. Then, it can be assumed that as the loading rate increases the onset of instability in the perturbation analysis - although this does not refer to the localized necking strain but to the diffuse necking strain as previously discussed - should also do. This suggest that a proper evaluation of the effect of loading rate on necking formation needs to jointly consider the damping effect of inertia on long wavelengths (with increasing loading rate), and the promotion of shorter wavelengths when the onset of instability is delayed (with increasing 


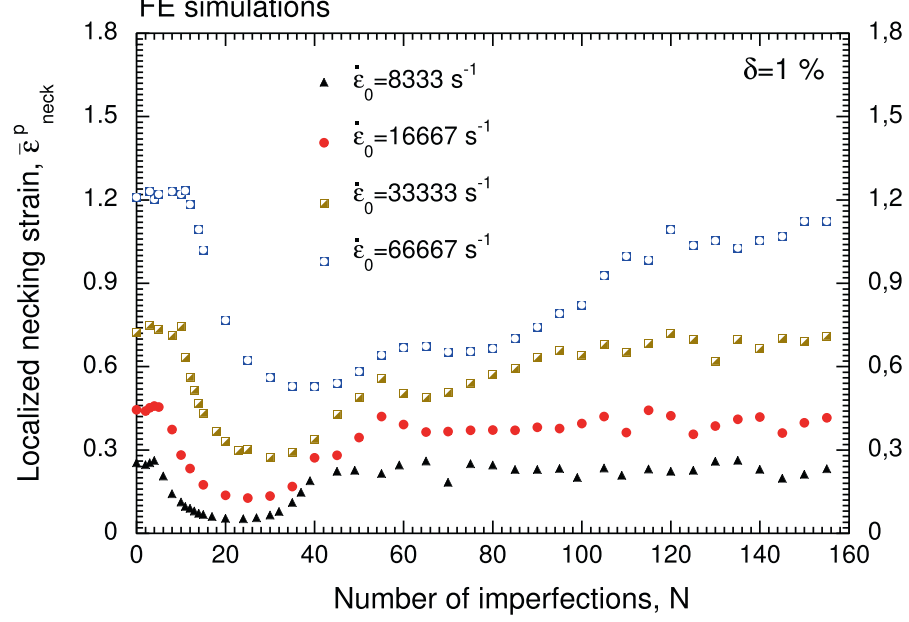

Fig. 7. Finite element results. Localized necking strain $\bar{\varepsilon}_{\text {neck }}^{p}$ upon the number of periodic imperfections $N$ for $\delta=1 \%$ and four different loading rates: $\dot{\varepsilon}_{0}=8333 \mathrm{~s}^{-1}, \dot{\varepsilon}_{0}=16667 \mathrm{~s}^{-1}, \dot{\varepsilon}_{0}=33333 \mathrm{~s}^{-1}$ and $\dot{\varepsilon}_{0}=66667 \mathrm{~s}^{-1}$.

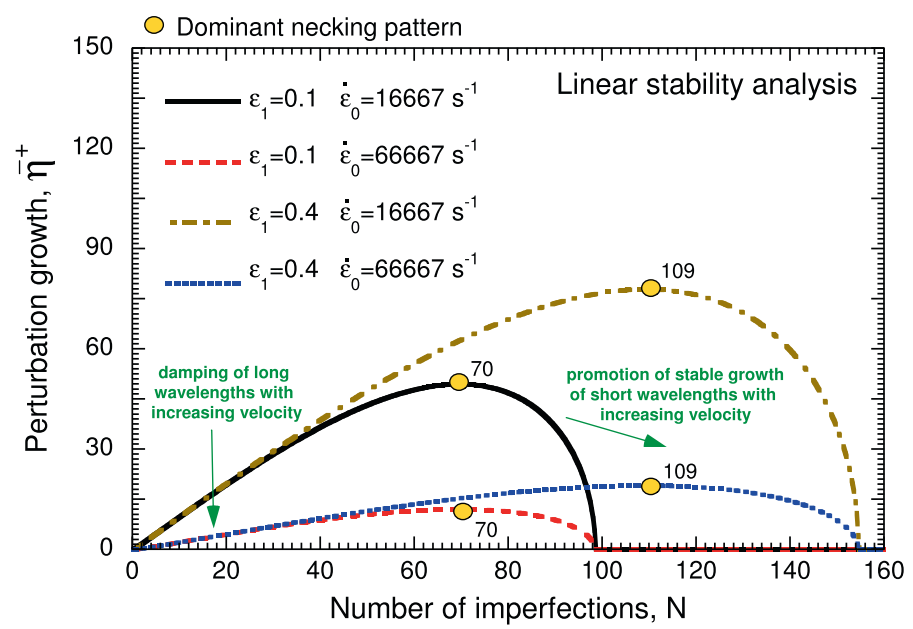

Fig. 8. Perturbation analysis results. Dimensionless perturbation growth $\bar{\eta}^{+}$upon the number of periodic imperfections $N$ for two different loading rates $\left(\dot{\varepsilon}_{0}=16667 \mathrm{~s}^{-1}, \dot{\varepsilon}_{0}=66667 \mathrm{~s}^{-1}\right)$ and two different background strains $\left(\varepsilon_{1}=0.1, \varepsilon_{1}=0.4\right)$.

loading rate); both effects are shown in Fig. 8. In consonance with Fig. 5 the upper bound of region I is shifted to shorter imperfections spacing with increasing loading rate since, as loading rate increases, the range of imperfection wavelengths that are damped by inertia is larger. Similarly, the lower bound of region III is shifted to shorter imperfections spacing with increasing loading rate since inertia delays the onset of necking and this promotes shorter wavelengths.

Moreover, the linear stability analysis predicts an increase in the dominant wavenumber with increasing loading velocity caused by its associated increase in the onset of the instability - background strain, as shown in Fig. 8. This behaviour agrees with the numerical computations results. However, the stability analysis does not capture the exact value of the dominant necking pattern predicted by the FE results. Two are the limitations of the stability analysis which cause this disagreement: (1) the wave propagation mechanisms which, below certain loading rates, play a role on the multiple necking process are not considered, see Mercier and Molinari (2004) and Rodríguez-Martínez et al. (2013b) for further details; (2) the uncertainty in the background strain value used to define the onset of instability (diffuse necking strain).

\subsection{Remarks: coupled imperfection-spacing/loading-rate analysis}

Previous results suggest that extinction of long wavelengths is favoured at high velocities and extinction of short wavelengths at low. This idea is reinforced by the finite element results shown in Fig. 9. This graph shows the 


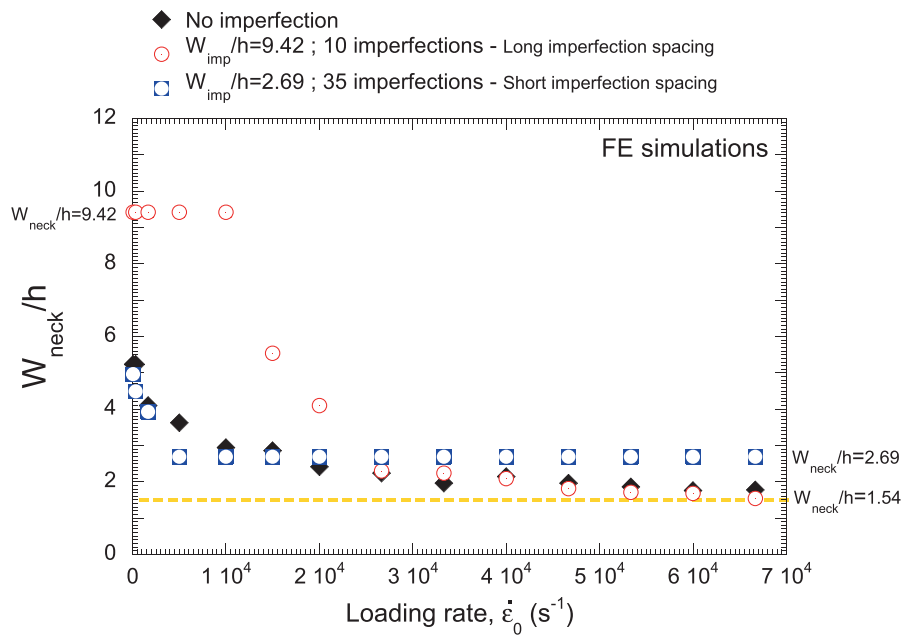

Fig. 9. Finite element results. Dimensionless neck spacing $W_{\text {neck }} / h$ as a function of the initial loading rate $\dot{\varepsilon}_{0}$ for three different numerical configurations: No imperfection $(\delta=0 \%)$, imperfection spacing $W_{\text {imp }} / h=9.42(\delta=1 \%)$ and imperfection spacing $W_{i m p} / h=2.69(\delta=1 \%)$.

dimensionless neck spacing $W_{\text {neck }} / h$ as a function of the initial loading rate $\dot{\varepsilon}_{0}$ for three different configurations:

1. Configuration A: No imperfection is introduced in the FE model. The localization is triggered by the numerical round-off which is sufficient to perturb the stress and strain fields (Rusinek and Zaera, 2007; Vadillo et al., 2012; Rodríguez-Martínez et al., 2013b). The necking pattern is rate dependent and shows deterministic nature. It should be noted that, with increasing loading rate, the dimensionless neck spacing $W_{\text {neck }} / h$ tends asymptotically to $W_{\text {neck }} / h \approx 1.5$. This value was identified by Rodríguez-Martínez et al. (2013b) as the critical wavelength which dictates the neck spacing in ductile rings expanded at very high strain rates. This critical wavelength was shown to be quite independent of the material properties but closely related to material inertia.

2. Configuration B: 10 imperfections are introduced in the FE model. This corresponds to an initial imperfection spacing $W_{\text {imp }} / h=9.42$. Let us denote this configuration as the long imperfection spacing configuration. Within the range of loading rates $\dot{\varepsilon}_{0} \lesssim 10000 \mathrm{~s}^{-1}$ the neck spacing coincides with the imperfection spacing. Inertia effects are not high enough to overcome the long wavelength imperfections, the necking process is stable. Within the range $10000 \mathrm{~s}^{-1} \lesssim \dot{\varepsilon}_{0} \lesssim 30000 \mathrm{~s}^{-1}$ neither the neck spacing coincides with the imperfections spacing nor the neck spacing coincides with the results of configuration A. Inertia only partially damps the long wavelength imperfections. Within the range $\dot{\varepsilon}_{0} \gtrsim 30000 \mathrm{~s}^{-1}$ the neck spacing agrees with the results obtained for configuration A. Inertia completely vanishes the effect of the imperfections distribution, the necking process is unstable.

3. Configuration C: 35 imperfections are introduced in the FE model. This corresponds to an initial imperfection spacing $W_{i m p} / h=2.69$. Let us denote this configuration as the short imperfection spacing configuration. Within the range of loading rates $\dot{\varepsilon}_{0} \lesssim 3000 \mathrm{~s}^{-1}$ the neck spacing coincides with the results obtained for configuration A. Stress multiaxiality effects completely vanish the imperfections distribution, the necking process is unstable. Within the range of loading velocities $3000 \mathrm{~s}^{-1} \lesssim \dot{\varepsilon}_{0} \lesssim 7000 \mathrm{~s}^{-1}$ neither the neck spacing matches with the imperfections spacing nor the neck spacing coincides with the results of configuration $A$. Within the range $\dot{\varepsilon}_{0} \gtrsim 7000 \mathrm{~s}^{-1}$ the neck spacing agrees with the imperfections spacing. Stress multiaxiality effects do not overcome the short wavelength imperfections, the necking process is stable.

\section{Discussion}

This paper examines the effects that inertia and stress multiaxiality have on the evolution of periodic geometric imperfections and necking inception in dynamically expanding ductile rings. In particular, the investigation has been focussed on demonstrating via finite element simulations and rationalizing via a linear stability analysis that inertia and stress multiaxiality can lead to the complete extinction of selected imperfection wavelengths. This is the main innovative feature of this work and provides further verification to the analytical considerations reported elsewhere (Fressengeas and Molinari, 1985; Fressengeas and Molinari, 1994; Shenoy and Freund, 1999; Mercier and Molinari, 2003; Mercier and Molinari, 2004). The main results of this investigation are schematically illustrated in Fig. 10, where $N_{I}^{I I}$ denotes the number of imperfections which defines the transition between regions I and II, $N_{\text {dom }}$ defines the number of imperfections which coincides with the number of necks determining the dominant necking pattern and $N_{I I}^{I I I}$ denotes the number of imperfections which defines the transition between regions II and III - note that the transition zones between regions where the necking process is partially stable and 
partially unstable are not included in the schematic representation.

If $N \leqslant N_{I}^{I I}$ the geometric imperfections introduced in the model do not act as neck precursor sites since they are completely suppressed due to inertia effects, Fig. 10(a). This behaviour gives way to the inception of an array of strain localization points whose distribution agrees with the necking pattern observed in absence of geometric imperfections. This necking pattern is deterministic and shows direct relation with the loading rate. Within this range of imperfection spacings, the localized necking strain $\bar{\varepsilon}_{\text {neck }}^{p}$ keeps largely constant (Fig. 10(b)). The value of $N_{I}^{I I}$ increases with loading velocity due to the proportional relation between strain rate and inertia. The value of $N_{I}^{I I}$ decreases with increasing imperfection amplitude due to the enhanced stress concentration caused by the associated cross section reduction (geometrical softening) which hinders the overcoming of the imperfections by inertia effects.

If $N_{I}^{I I} \leqslant N \leqslant N_{I I}^{I I I}$ the number of necks incepted coincides with the number of imperfections introduced in the model, Fig. 10(a). Furthermore, each imperfection leads to the nucleation of a single neck. Within this range of imperfections spacing, the localized necking strain $\bar{\varepsilon}_{\text {neck }}^{p}$ largely

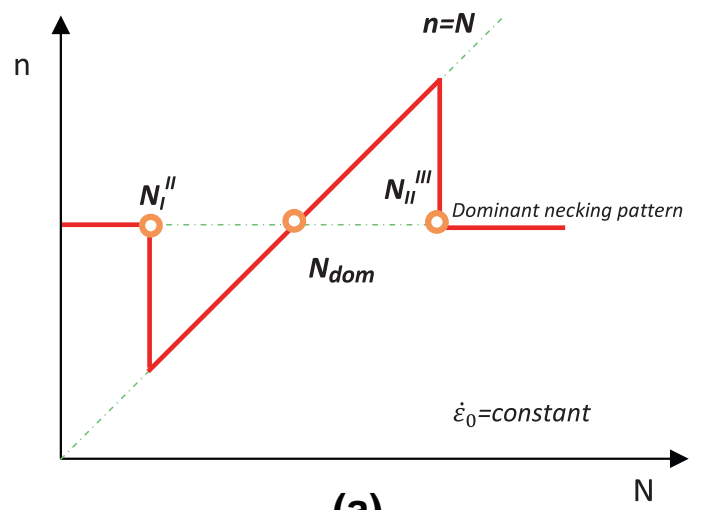

(a)

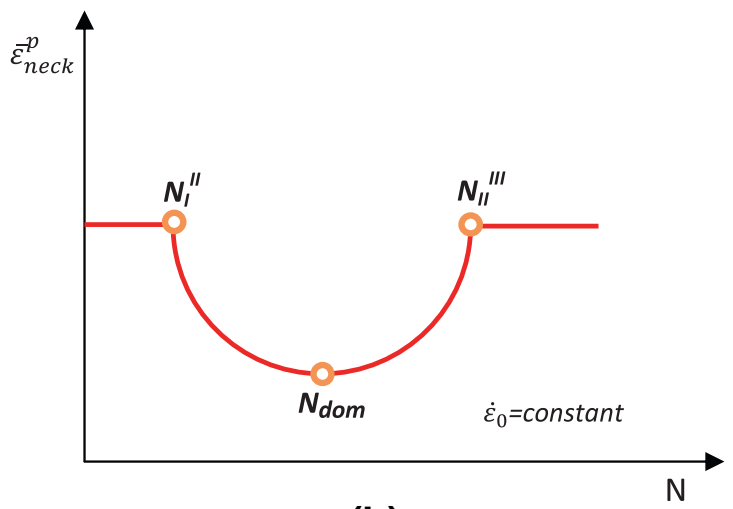

(b)

Fig. 10. Representative schemes. (a) Number of necks $n$ upon the number of imperfections $N$. (b) Localized necking strain $\bar{\varepsilon}_{\text {neck }}^{p}$ upon the number of imperfections $N$. varies with the number of imperfections introduced in the model (Fig. 10(b)). Namely, $\bar{\varepsilon}_{\text {neck }}^{p}$ decreases with $N$ for imperfection spacings larger than that defined by the dominant necking pattern and increases with $N$ for imperfection spacings smaller than that defined by the dominant necking pattern. The number of imperfections which matches with the dominant necking pattern determines the minimum value of $\bar{\varepsilon}_{\text {neck }}^{p}$, i.e. the minimum investment of energy required for necking formation.

If $N \geqslant N_{I I}^{I I I}$ the geometric imperfections introduced in the model do not act as neck precursor sites since they are completely extinguished due to stress multiaxiality effects, Fig. 10(a). This behaviour gives way to the inception of an array of strain localization points whose distribution agrees with the necking pattern observed in absence of geometric imperfections. Within this range of imperfection spacings the localized necking strain $\bar{\varepsilon}_{\text {neck }}^{p}$ keeps largely constant (Fig. 10(b)). The value of $N_{I I}^{I I I}$ increases with loading velocity since strain rate retards necking formation which promotes the development of shorter wavelengths as shown schematically in Fig. 11. The value of $N_{I I}^{I I I}$ increases with increasing imperfection amplitude due to the enhanced stress concentration caused by the associated cross section reduction which hinders the overcoming of the imperfections by stress multiaxiality effects. The discussed effect of loading rate in the variation of the position of $N_{I}^{I I}$ and $N_{I I}^{I I I}$ (moving both lower and upper bounds to higher values of $N$ with increasing $\dot{\varepsilon}_{0}$ ) is represented schematically in Fig. 12.

Fig. 12 also shows the role played by loading velocity on the number of necks incepted for three different imperfection spacings. These imperfection spacings are assumed analogous to those discussed in Fig. 9. For a material without geometrical imperfections, the necking spacing is coincident with the dominant one and decreases with loading rate. Considering the longer imperfection wavelength, the neck spacing matches the imperfection spacing for the lower loading rate $\dot{\varepsilon}_{0(1)}$ and coincides with the dominant spacing for the higher $\dot{\varepsilon}_{0(2)}$. Considering the short imperfection wavelength, the neck spacing matches the dominant spacing for the lower loading rate $\dot{\varepsilon}_{0(1)}$ and coincides with the imperfection spacing for the higher $\dot{\varepsilon}_{0(2)}$; being this neck spacing larger than that determined by the dominant one in agreement with Fig. 9.

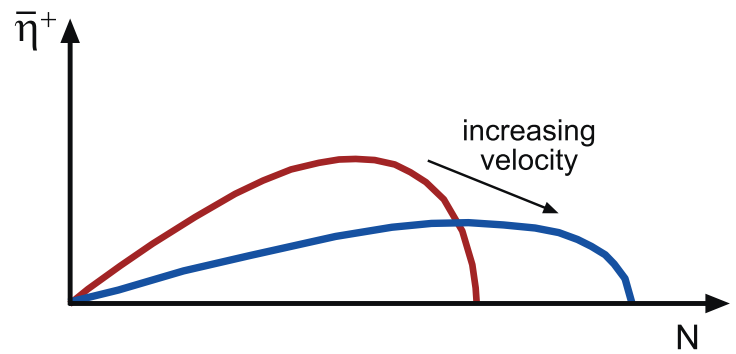

Fig. 11. Schematic representation of the dimensionless perturbation growth $\bar{\eta}^{+}$upon the number of imperfections $N$ for increasing velocity (taking into account the associated increase in background strain). 


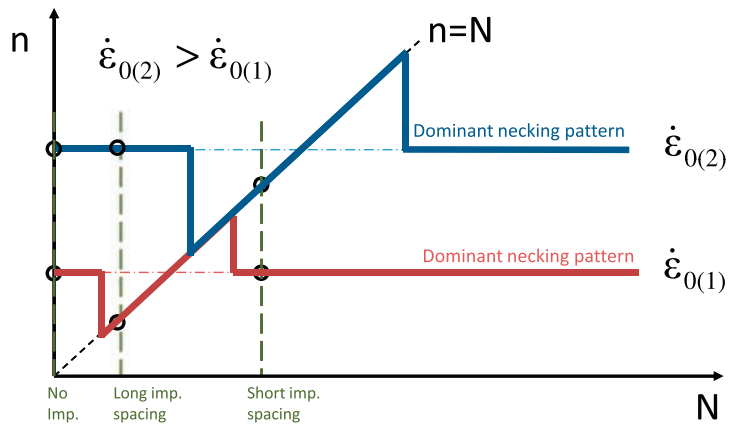

Fig. 12. Representative scheme: Number of necks $n$ as a function of number of imperfections $N$ for two different initial loading rates $\dot{\varepsilon}_{0}$.

\section{Concluding remarks}

From this analysis emerges a direct connection between geometric imperfections, loading velocity and necking strain which determines the multiple necking formation in ductile rings expanded at high strain rates. Understanding of this relationship has main importance since the presence of manufacturing or material defects in the form of surface or geometric imperfections is inherent to any structural element. The main conclusions are as follows:

1. In agreement with stability analyses, finite element computations corroborate that sufficiently long wavelength imperfections are completely suppressed by inertia and sufficiently short wavelength imperfections are completely suppressed by stress multiaxiality effects.

2. It has been demonstrated that the loading velocity plays a major role on the extinction of imperfection wavelengths. Namely, the extinction of long imperfection wavelengths is favoured at high loading velocities and extinction of short imperfection wavelengths at low. These results have been rationalized based on a linear stability analysis.

3. It has been shown that the necking strain, the reference variable in determining the capacity of ductile materials for absorbing energy under dynamic loadings, is highly dependent on the imperfections pattern. In particular, it has been detected that the maximum necking strain is registered for the loading cases in which the imperfections distribution is completely extinguished either by inertia or stress multiaxiality effects. Furthermore, there exists a dominant necking pattern which emerges when the geometric imperfections are vanished. This dominant neck spacing shows direct relation with the loading rate.

\section{Acknowledgements}

The authors are indebted to the Ministerio de Ciencia e Innovación de España (Projects DPI/2011-24068 and DPI/ 2011-23191) for the financial support.
The authors express sincere gratitude to Professor Alain Molinari for helpful discussions on multiple necking problems.

\section{References}

Altynova, M., Hu, X., Daehn, G.S., 1996. Increased ductility in high velocity electromagnetic ring expansion. Metallurgical Transactions A 27, 1837-1844.

Bridgman, P.W., 1952. Plastic wave in a wire extended by an impact load. Scientific Papers. Mechanics of Solids, vol. 1. McGraw-Hill Book Company, Inc., New York, pp. 9-37.

Elek, P., Jaramaz, S., 2005. Modeling of fragmentation of rapidly expanding cylinders. Theoretical and Applied Mechanics 32, 113-130.

Elek, P., Jaramaz, S., 2008. Fragment size distribution in dynamic fragmentation: geometric probability approach. FME Transactions 36, 59-65.

Fressengeas, C., Molinari, A., 1985. Inertia and thermal effects on the localization of plastic flow. Acta Metallurgica 33, 387-396.

Fressengeas, C., Molinari, A., 1994. Fragmentation of rapidly stretching sheets. European Journal of Mechanics A/Solids 13, 251-268.

Fyfe, I.M., Rajendran, A.M., 1980. Dynamic pre-strain and inertia effects on the fracture of metals. Journal of the Mechanics and Physics of Solids $28,17-26$.

Goto, D., Becker, R., Orzechowski, T., Springer, H., Sunwoo, A., Syn, C., 2008. Investigation of the fracture and fragmentation of explosively driven rings and cylinders. International Journal of Impact Engineering 35, 1547-1556.

Gourdin, W.H., 1989. Analysis and assessment of electromagnetic ring expansion as a high-strain-rate test. Journal of Applied Physics 65, 411-422.

Grady, D., 1981. Fragmentation of solids under impulsive stress loading. Journal of Geophysical Research 86, 1047-1054.

Grady, D.E., Benson, D.A., 1983. Fragmentation of metal rings by electromagnetic loading. Experimental Mechanics 12, 393-400.

Grady, D.E., Olsen, M.L., 2003. A statistics and energy based theory of dynamic fragmentation. International Journal of Impact Engineering 29, 293-306.

Han, J.B., Tvergaard, V., 1995. Effect of inertia on the necking behaviour of ring specimens under rapid axial expansion. European Journal of Mechanics A/Solids 14, 287-307.

Hill, R., Hutchinson, J.W., 1975. Bifurcation phenomena in the plane tension test. Journal of the Mechanics and Physics of Solids 23, 239264.

Hiroe, T., Fujiwara, K., Hata, H., Takahashi, H., 2008. Deformation and fragmentation behaviour of exploded metal cylinders and the effects of wall materials, configuration, explosive energy and initiated locations. International Journal of Impact Engineering 35, 1578-1586.

$\mathrm{Hu}$, X., Daehn, G.S., 1996. Effect of velocity on flow localization in tension. Acta Materialia 44, 1021-1033.

Janiszewski, J., 2012. Ductility of selected metals under electromagnetic ring test loading conditions. International Journal of Solids and Structures 49, 1001-1008.

Juanicotena, A., 1998. Etude théorique et expérimentale du comportement viscoplastique des matériaux aux grandes déformations et grandes vitesses de déformation. Ph.D. thesis, Université de Metz and Commissariat à l'Energie Atomique.

Kipp, M., Grady, D., 1985. Dynamic fracture growth and interaction in one dimension. Journal of the Mechanics and Physics of Solids 33, 399415.

Knoche, P., Needleman, A., 1993. The effect of size on the ductility of dynamically loaded tensile bars. European Journal of Mechanics A/ Solids 12, 586-601.

Mercier, S., Molinari, A., 2003. Predictions of bifurcations and instabilities during dynamic extensions. International Journal of Solids and Structures 40, 1995-2016.

Mercier, S., Molinari, A., 2004. Analysis of multiple necking in rings under rapid radial expansion. International Journal of Impact Engineering 4, 403-419.

Mercier, S., Granier, N., Molinari, A., Llorca, F., Buy, F., 2010. Multiple necking during the dynamic expansion of hemispherical metallic shells, from experiments to modelling. Journal of the Mechanics and Physics of Solids 58, 955-982.

Mott, N. F., 1947. Fragmentation of shell cases. Series A. Proceedings of the Royal Society, London, pp. 300-308.

Needleman, A., 1991. The effect of material inertia on neck development. In: Yang, W.H. (Ed.), Topics in Plasticity. AM Press, Ann Arbor, MI, pp. 151-160. 
Niordson, F.L., 1965. A unit for testing materials at high strain rates. Experimental Mechanics 5, 29-32.

Plěsek, J., Kolman, R., Gabriel, D., 2010. Dispersion errors of finite element discretizations in elastodynamics. Computational and Technology Reviews 1, 251-279.

Rodríguez-Martínez, J.A., Rittel, D., Zaera, R., Osovski, S., 2013a. Finite element analysis of AISI 304 steel sheets subjected to dynamic tension: the effects of martensitic transformation and plastic strain development on flow localization. International Journal of Impact Engineering 54, 206-216.

Rodríguez-Martínez, J.A., Vadillo, G., Fernández-Sáez, J., Molinari, A., 2013b. Identification of the critical wavelength responsible for the fragmentation of ductile rings expanding at very high strain rates. Journal of the Mechanics and Physics of Solids. http://dx.doi.org/ 10.1016/j.jmps.2013.02.003.

Rusinek, A., Zaera, R., 2007. Finite element simulation of steel ring fragmentation under radial expansion. International Journal of Impact Engineering 34, 799-822.

Shenoy, V.B., Freund, L.B., 1999. Necking bifurcations during high strain rate extension. Journal of the Mechanics and Physics of Solids 47, 2209-2233.

Simulia, 2010. ABAQUS/Explicit User's Manual, version 6.10 Edition. Dassault Systèmes, Providence, USA.

Sørensen, N.J., Freund, L.B., 1998. Dynamic bifurcation during high-rate planar extension of a thin rectangular block. European Journal of Mechanics A/Solids 17, 709-724.

Sørensen, N.J., Freund, L.B., 2000. Unstable neck formation in a ductile ring subjected to impulsive radial loading. International Journal of Solids and Structures 37, 2265-2283.

Triantafyllidis, N., Waldenmyer, J.R., 2004. Onset of necking in electromagnetically formed rings. Journal of Mechanics and Physics of Solids $52,2127-2148$.
Tuğcu, P., 1996. Inertial effects in ductile failure of cylindrical tubes under internal pressure. International Journal of Impact Engineering 18, 539-563.

Tuğcu, P., 2003. Instability and ductile failure of thin cylindrical tubes under internal pressure impact. International Journal of Impact Engineering 28, 183-205.

Vadillo, G., Rodríguez-Martínez, J.A., Fernández-Sáez, J., 2012. On the interplay between strain rate and strain rate sensitivity on flow localization in the dynamic expansion of ductile rings. International Journal of Solids and Structures 49, 481-491.

Walsh, J.M., 1984. Plastic instability and particulation in stretching metals jets. Journal of Applied Physics 56, 1997-2006.

Wesenberg, D., Sagartz, M., 1977. Dynamic fracture of 6061-T6 aluminium cylinders. Journal of Applied Mechanics 44, 643-646.

Xue, Z., Vaziri, A., Hutchinson, J.W., 2008. Material aspects of dynamic neck retardation. Journal of the Mechanics and Physics of Solids 56, 93-113.

Zhang, H., Ravi-Chandar, K., 2006. On the dynamics of necking and fragmentation - I. Real-time and post-mortem observations in $\mathrm{Al}$ 6061-O. International Journal of Fracture 142, 183-217.

Zhang, H., Ravi-Chandar, K., 2010. On the dynamics of localization and fragmentation-IV. Expansion of al 6061-O tubes. International Journal of Fracture 163, 41-65.

Zhang, L., Jin, X., He, H., 1999. Prediction of fragment number and size distribution in dynamic fracture. Journal of Physics D: Applied Physics $32,612-615$.

Zhou, F., Molinari, J.F., Ramesh, K.T., 2006. An elasto-visco-plastic analysis of ductile expanding ring. International Journal of Impact Engineering 33, 880-891.

Zukas, J.A., Scheffer, D.R., 2000. Practical aspects of numerical simulations of dynamic events: effects of meshing. International Journal of Impact Engineering 24, 925-945. 